\title{
Depercolation of aggregates upon polymer grafting in simplified industrial nanocomposites studied with dielectric spectroscopy
}

\author{
Guilhem P. Baeza ${ }^{1,2}$, Julian Oberdisse ${ }^{1}$, Angel Alegria ${ }^{3}$, Kay Saalwächter ${ }^{4}$, Marc Couty ${ }^{2}$ and \\ Anne-Caroline Genix ${ }^{1 *}$ \\ ${ }^{1}$ Laboratoire Charles Coulomb (L2C), UMR 5221 CNRS-Université de Montpellier, F-34095 Montpellier, France. \\ ${ }^{2}$ Manufacture Française des Pneumatiques MICHELIN, Site de Ladoux, 23 place des Carmes Déchaux, F-63 040 Clermont- \\ Ferrand, Cedex 9, France \\ ${ }^{3}$ Departamento de Física de Materiales y Centro de Física de Materiales CSIC-UPV/EHU, Universidad del País Vasco \\ UPV/EHU, Paseo Manuel de Lardizabal 5, E-20018 San Sebastian, Spain \\ ${ }^{4}$ Institut für Physik, Martin-Luther Universität Halle Wittenberg, 06099 Halle (Saale), Germany \\ * acgenix@univ-montp2.fr - Phone: 0033467144140 - Fax: 0033467144637
}

\begin{abstract}
The dynamics of polymer and filler in simplified industrial silica-styrene-butadiene nanocomposites (silica Zeosil $1165 \mathrm{MP}$, volume fraction $0-21 \% \mathrm{v}$ ) have been studied with broadband dielectric spectroscopy (BDS) and nuclear magnetic resonance (NMR). The fraction of graftable matrix chains was varied from $0-100 \% \mathrm{D} 3$. The introduction of silica nanoparticles is shown to leave the segmental relaxation unaffected, an observation confirmed by the measurement of only a thin (some Angstroms thick) immobilized layer by NMR. The low-frequency measurements are resolved in two distinct dielectric Maxwell-Wagner-Sillars (MWS) processes of different behavior with respect to changes of large-scale silica structures induced by variations of filler fraction and grafting. It is found that increasing grafting leaves the first MWS-process unaffected, while it decreases the strength of the (slower) second MWS by about a decade. At constant silica volume fraction, this indicates depercolation of the filler, thereby providing a microscopic explanation of the evolution of rheological reinforcement. The sensitivity to large-scale reorganizations together with a characterization of local polymer dynamics provides insight over many length- and time-scales into structure and dynamics of nanocomposites, and thus the physical origin of the reinforcement effect.
\end{abstract}




\section{INTRODUCTION}

Nanocomposites made of nanometric filler particles embedded in a viscoelastic polymer matrix may have outstanding macroscopic properties, among which one can cite mechanical, electrical, or optical ones.[1-4] This broad range makes such systems suitable candidates for applications in very different fields, from car tires to, e.g., biological tissue engineering[5]. Microscopic understanding is necessary if one wishes to tune macroscopic properties of interest, like for instance the optimization of (non-)linear dynamic mechanical properties of car tire material in order to reduce rolling resistance, and thus fuel consumption and $\mathrm{CO}_{2}$ production. The basis of macroscopic properties is the microstructure, i.e. the dispersion state of the nanoparticles (NPs), which may be individually dispersed, organized in small aggregates, or form sample-spanning networks of percolated particles or aggregates.[6-10] The microstructure sets the frame for the dynamical properties, which themselves control many macroscopic properties, like, e.g., the time-dependent reaction of samples to mechanical stress which is often based on structural reorganization $[11,12]$.

The local segmental dynamics in nanocomposites has been studied by several groups in the past [13-22]. Polymer in contact with NP surfaces may form immobilized, sometimes referred to as "glassy", layers which are mainly detected by NMR [13, 23]. On larger scales, at high temperature (above the glass transition), the occurrence of a Maxwell-Wagner-Sillars (MWS) process has been reported in several composite systems using broadband dielectric spectroscopy (BDS) [21, 24-26]. MWS processes are related to polarization effects due to charge carrier diffusion through the different phases of the nanocomposites. These effects result in the trapping and accumulation of charges at the polymer/silica interface. Charges can diffuse on the surface of individual NPs within aggregates, or on a larger scale on the surface of aggregates [27], processes which have been described theoretically by the interlayer model (ILM) $[28,29]$. The ionic conductivity has also been studied over the whole frequency range 
$[27,30]$. In a biphasic material, one may note that the measured conductivity is a global observable, which is related to the polymer, the filler via its surface conductivity, and to the spatial arrangement of the filler which may form conductive pathways of various sizes within the sample.

The dynamical properties of nanocomposites as measured by BDS and NMR have only rarely been compared to macroscopic rheological properties. A correlation has been described in the literature for carbon-black filled elastomers [27, 30] and clay nanocomposites [25]. It is unclear, however, if the reinforcement of mechanical properties is dominated by local dynamical heterogeneities bridging NPs [31-33] or large-scale filler structure [34, 35]. As far as we know, it is a novelty to study this question using dielectric spectroscopy of silica nanocomposites with multi-scale structure.

Recently, we have contributed to the understanding of the multi-scale structure of silicastyrene-butadiene (SB) simplified industrial nanocomposites using a combination of smallangle scattering, electron microscopy, and numerical simulations.[36-39] Here simplified refers to the absence of any crosslinker and related catalysts. We have built a global model describing structural data where primary silica beads (typical size $17 \mathrm{~nm}$ ) are organized within small aggregates (typical size $80 \mathrm{~nm}$, aggregation number about 50, giving an internal volume fraction, i.e., compacity, of the order of $35 \%$ ), which themselves fill large-scale fractal branches (typical width $150 \mathrm{~nm}$ ). The solid-phase mixing protocol (see SI) applied to industrial silica pellets result in a broad aggregate size distribution of polydispersity $30 \%$. In a first paper focused on dynamics, we have characterized by BDS a new structure-related hightemperature dielectric process termed MWS2, as it occurs in addition to the previously observed MWS process [40]. It takes place across polymer between aggregates as found by an analysis of activation energies [40]. Under $\Phi_{\mathrm{si}}$ variation which induces aggregate percolation 
(network formation), a strong increase of the temperature-independent dielectric strength of MWS2 was found around the mechanical percolation threshold of $12 \% \mathrm{v}$.

The key question of this second article on dynamics is to understand the relationship between structure and dynamical processes by now varying principally the amount of graftable SB chains (\%D3) in order to control the filler structure [37]. Indeed, local dynamics as observed by NMR has been correlated with rheology [32] but the question of the relative importance of large-scale structure with respect to local connections (bridges) through immobilized layers remains open. We therefore focus in the first part of this article on the local scale, and in particular on segmental relaxation investigated by BDS and NMR, as a function of silica content and grafting. Then, the impact of polymer grafting on interfacial dynamics of largescale silica aggregate depercolation (network destruction) is studied. Finally, both local and large-scale dynamical properties are linked to rheology.

\section{EXPERIMENTAL SECTION}

2.1 Materials. Details are given in ref $[36,37]$. The polymer matrix is made of styrenebutadiene (SB) random copolymers, with two types of chains of molecular mass $140 \mathrm{~kg} \cdot \mathrm{mol}^{-1}$ $(\mathrm{PI}=1.07)$ as determined by size exclusion chromatography. Chains are either linear unmodified SB chains, or bear a single silanol end-function making the chains graftable onto the silica surface by condensation. These functionalized chains are denoted D3, and the matrix composition is referred to by the percentage of D3 chains (\%D3). Both types of chains have been purpose-synthesized by Michelin. The silica pellets (Zeosil 1165 MP from Solvay) have a nominal specific surface of $160 \mathrm{~m}^{2} / \mathrm{g}$, and the size distribution of the nanoparticles obeys a log-normal law with $\mathrm{R}_{0}=8.55 \mathrm{~nm}, \sigma=27 \%$.[36] Nanocomposite formulation is the same as before $[36,37]$ and is detailed in the SI. Real silica volume fractions in nanocomposites have been measured by thermogravimetric analysis (Q500, TA Instruments) and lie in the range $\Phi_{\mathrm{si}}$ 
$=8.4 \% \mathrm{v}-21.1 \% \mathrm{v}$ (i.e., between 21 and $63 \mathrm{phr}$ ). The glass-transition temperature was determined using differential scanning calorimetry (Q2000 TMDSC, TA Instruments) in the modulated mode with a heating rate of $3 \mathrm{~K} / \mathrm{min}$. For the filled samples, $\mathrm{T}_{\mathrm{g}}$ shows no significant variation with silica content and $\% \mathrm{D} 3$ as compared to the pure polymer matrix: $\mathrm{T}_{\mathrm{g}}$ $=-37 \pm 0.8^{\circ} \mathrm{C}$.

2.2 Broadband dielectric spectroscopy (BDS). A broadband high-resolution dielectric spectrometer (Novocontrol Alpha) was used to measure the complex dielectric permittivity $\varepsilon^{*}(\omega)=\varepsilon^{\prime}(\omega)-\mathrm{i} \varepsilon^{\prime}(\omega)$ in the frequency range from $\mathrm{f}=10^{-2}$ to $10^{7} \mathrm{~Hz}(\omega=2 \pi \mathrm{f})$ of samples prepared as before [40]. After 20 minutes equilibration at $60^{\circ} \mathrm{C}$ in the $\mathrm{BDS}$ cryostat (under nitrogen atmosphere), isothermal frequency measurements have been performed with temperature stability better than $0.1 \mathrm{~K}$. Two series of nanocomposites have been investigated: samples with matrix composition fixed to $50 \% \mathrm{D} 3$ and $\Phi_{\text {si }}$ ranging from 8.4 to $21.1 \% \mathrm{v}$, and samples with fixed $\Phi_{\mathrm{si}}(\approx 8.5 \% \mathrm{v})$ and $\% \mathrm{D} 3$ varying from 0 to $100 \%$. For all samples, the temperature sequence starts from $333 \mathrm{~K}$ down to $153 \mathrm{~K}$. Afterwards, the high-T range between 333 and $363 \mathrm{~K}$ has been explored.

BDS fitting. Data treatment was carried out simultaneously on $\varepsilon$ ' and $\varepsilon$ ' using for each temperature the combination of a purely dissipative dc-conductivity term and one HavriliakNegami $(\mathrm{HN})$ for each dipolar relaxation or MWS process present in the frequency window

$$
\varepsilon^{*}(\omega)=\varepsilon_{\infty}+\sum_{j} \frac{\Delta \varepsilon_{j}}{\left[1+\left(i \omega \tau_{H N_{j}}\right)^{\gamma_{j}}\right]^{\delta_{j}}}-i \frac{\sigma_{d c}}{\varepsilon_{0} \omega}
$$

where $\gamma_{j}$ and $\delta_{j}$ are two shape parameters ranging between 0 and 1 , and describing the symmetric and asymmetric broadening of the $\mathrm{j}^{\text {th }}$ process, respectively. $\Delta \varepsilon_{\mathrm{j}}$ is the dielectric relaxation strength corresponding to the difference between the unrelaxed and relaxed values 
of the dielectric constant, and $\tau_{\mathrm{HNj}}$ is the characteristic relaxation time. In our approach, we systematically reduced the number of free parameters in order to extract reliable information, in particular on dielectric strengths and time scales for overlapping processes. A first fit of the data has been performed with all variables let free in the fit function to identify possible trends with temperature for the $\mathrm{HN}$ shape parameters. Then, according to the results, these parameters were forced to follow a linear (possibly constant) behavior (see SI).

Dielectric processes. Processes in the neat SB matrix (50\%D3) have been investigated, i.e., the $\alpha$-relaxation linked to the glass transition and the secondary $\beta$-relaxation which has been related to local motions within the butadiene units [41]. In nanocomposites, two additional high-temperature dielectric (MWS1 and MWS2) processes at time scales slower than the $\alpha$ relaxation have been identified [40]. The HN parameters for both processes - shape parameters and dielectric strength - were found to hardly vary with $\mathrm{T}$, and have been fixed to their average value in the final fitting procedure.[40] We obtain a broad distribution of relaxation times $\left(\gamma_{\mathrm{MWS} 1}=0.42\right.$ and $\left.\gamma_{\mathrm{MWS} 2}=0.55\right)$, in agreement with structurally polydisperse systems [36]. Also, the secondary $\beta$-relaxation in pure polymer is masked in nanocomposites by the more intense $\beta$ '-relaxation, which is also slightly shifted in frequency. A summary plot with all contributions appearing in $\varepsilon^{\prime}(\omega)$ and $\varepsilon^{\prime \prime}(\omega)$ over a temperature window from $173 \mathrm{~K}$ to $323 \mathrm{~K}$, and fit details are given in the SI. The $\beta$ '-process is well-known in the silica-SB nanocomposites, where it has been assigned to localized fluctuations of water molecules at the silica surface $[19,42]$. A complete analysis of its evolution with $\Phi_{\text {si }}$ and $\% \mathrm{D} 3$ is given in the SI.

Special care was given to the description of the $\alpha$-relaxation in the nanocomposites as it was found to overlap with the much more intense MWS1-process. In this case, shape parameters $\left(\gamma_{\alpha}\right.$ and $\left.\delta_{\alpha}\right)$ were imposed to the same values as in the pure matrix (see SI). To account 
properly for its relaxation strength in the nanocomposite, we have considered the filler effect on the local field according to ref [43], using a simple model for two-phase heterogeneous materials with 2.5 and 5.5 for the dielectric permittivity of SB and silica ${ }^{36,37}$, respectively. The characteristic relaxation time was consequently left as the only free fit parameter for this process.

Temperature dependence. For each dielectric process, the relaxation time corresponding to the frequency of maximal loss $\tau_{\max }=\left(2 \pi \mathrm{f}_{\max }\right)^{-1}$ is a convenient parameter which can be read off from the graph $\varepsilon$ "(f) or determined from the fit parameters. These times are plotted in Figure 1 for the matrix processes together with all other processes observed in nanocomposites.

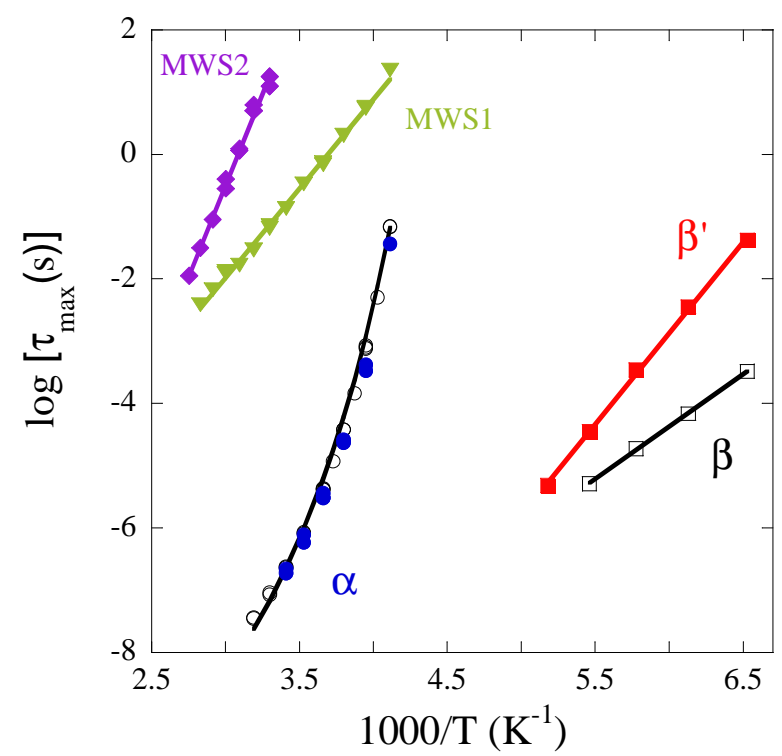

Figure 1: Complete relaxation map for the neat SB (black empty symbols) and a nanocomposite filled with $8.4 \% \mathrm{v}$ of silica (plain symbols with different colors for the different processes investigated, see the dielectric spectra and other fit parameters in SI). The solid lines through data points are fits to the VFT equation for the $\alpha$-relaxation and to the Arrhenius equation for the others.

For all processes except the segmental relaxation, the temperature dependence of the relaxation times in Figure 1 is described by the Arrhenius equation 


$$
\tau(T)=\tau_{0} \exp \left(\frac{E}{k_{B} T}\right)
$$

where $\mathrm{E}$ is the activation energy, $\mathrm{k}_{\mathrm{B}}$ the Boltzmann constant and $\tau_{0}$ the high-temperature limit associated to vibrational times. In contrast, the temperature dependence of the $\alpha$-relaxation times display a non-Arrhenius behavior, which can be well described by the Vogel-FulcherTammann equation [43]

$$
\tau(\mathrm{T})=\tau_{0} \exp \left(\frac{\mathrm{DT}_{0}}{\mathrm{~T}-\mathrm{T}_{0}}\right)
$$

where $T_{0}$ is the temperature at which $\tau$ would diverge and $\mathrm{D}$ a coefficient related to the fragility concept [44]. Fitting the data in Figure 1 using eq(2a) led to $E_{\beta}=32 \mathrm{~kJ} / \mathrm{mol}$ in good agreement with ref [41] $\left(\mathrm{E}_{\beta}=34.3 \pm 1.3 \mathrm{~kJ} / \mathrm{mol}\right.$ independent of the styrene content $)$. For the $\alpha$-relaxation, we obtained $\tau_{0}=10^{-12.8} \mathrm{~s}, \mathrm{~T}_{0}=187 \mathrm{~K}$ and $\mathrm{D}=8.1$ from our data (the corresponding fragility value is $\mathrm{m}=76$ ), in good agreement with the work of Ferry [45] and Cerveny et al [41] on close SB samples $(m=69 \pm 3)$. The temperature value corresponding to $\tau_{\alpha}=100 \mathrm{~s}$ gives an estimation of the glass-transition temperature, $\mathrm{T}_{\mathrm{g}}(100 \mathrm{~s})=231.5 \mathrm{~K}$, to be compared with the calorimetric value $\mathrm{T}_{\mathrm{g}}=236 \mathrm{~K}$. Turning to the silica related processes, they all follow an Arrhenius temperature-dependence with $\mathrm{E}_{\beta},=56 \mathrm{~kJ} / \mathrm{mol}, \mathrm{E}_{\mathrm{MwS} 1}=55 \mathrm{~kJ} / \mathrm{mol}$ and $\mathrm{E}_{\mathrm{MwS} 2}=113 \mathrm{~kJ} / \mathrm{mol}$, which will also be discussed below.

2.3 Low field NMR. Proton NMR experiments were performed using a Bruker Minispec mq20 with a proton resonance frequency of $20 \mathrm{MHz}(0.5$ Tesla). Free induction decay (FID) and magic sandwich echo FID (MSE-FID) signals, which are sensitive to molecular motions, were measured between 10 and $70^{\circ} \mathrm{C}$ in order to extract a solid-like signal fraction in the SB nanocomposites using a recycle delay of $2 \mathrm{~s}$. The MSE-FID allows refocussing the FID signal and thus accesses the whole decay including the very first part, which is not available in simple FID due to the spectrometer dead time. The used methods have been reviewed in ref 
[46], and details about the sequences can be found in refs [14, 47]. The signal of the nanocomposites can be decomposed in two contributions: a fast decay corresponding to the response of the rigid part and a slow decay associated to the mobile part. The data up to $t=$ $200 \mu$ s have then been fitted to [32]

$$
\frac{M(t)}{M_{0}}=a_{\text {rig }} \exp \left(-\left(t / \tau_{\text {rig }}\right)^{2}\right)+\left(1-a_{\text {rig }}\right) \exp \left(-\left(t / \tau_{\text {mobil }}\right)^{\beta}\right)
$$

where $a_{\text {rig }}$ is the fraction of rigid polymer. In order to avoid (small) systematic errors related to signal loss during the MSE, we used the unfiltered signal (FID) in all cases except at $10^{\circ} \mathrm{C}$ where the filtered signal (MSE-FID) led to more stable fits. The value for the short decay time constant $\tau_{\text {rig }}$ was determined independently from a double-quantum (DQ) filtered signal [47], where the mobile part is almost entirely suppressed. Within error bars, it was found not to vary significantly with $\Phi_{\text {si }}$ (or $\left.\% \mathrm{D} 3\right)$ and the temperature: $\tau_{\text {rig }}=(30 \pm 3) \mu \mathrm{s}$. It has been fixed to this value in eq 3. It is noted that the main effect of softening with temperature should be an increase of $\tau_{\text {rig }}$ at constant fraction accompanied by non-trivial changes in the shape of the relaxation (decay) function.[14] Owing to the small overall fraction, because of which fitting is restricted to a two-component model, the change in relaxation behavior is thus qualitatively reflected in the apparent decrease of the rigid fraction.

2.4 Rheology. The rheological response in the linear regime expressed by the storage modulus, $\mathrm{G}^{\prime}(\omega)$, was obtained with a stress-controlled rheometer AR 2000, used in the straincontrolled mode in plate-plate geometry $(20 \mathrm{~mm}$ diameter). Isothermal frequency sweeps at fixed low deformation level $(\gamma=0.1 \%)$ were performed in the temperature range from $10^{\circ} \mathrm{C}$ to $80^{\circ} \mathrm{C}$ allowing to cover a large frequency range from $10^{-2}$ to $10^{4} \mathrm{rad} / \mathrm{s}$ using the principle of time-temperature superposition for a reference temperature of $50^{\circ} \mathrm{C}$. 


\section{Results}

\subsection{Segmental relaxation in nanocomposites}

The $\alpha$-relaxation is detected in the range from 243 and $293 \mathrm{~K}$ in nanocomposites with various silica fractions and $\% \mathrm{D} 3$. The dielectric response at $273 \mathrm{~K}$ is shown in Figure 2 where one can also see the MWS1-process at lower frequency. The fit function by means of eq 1 (see SI for the detailed fit contributions) is also included. Note that, for all samples, the description is possible using highly constrained parameters. Namely, the shape and the dielectric strength of the $\alpha$-process can be fixed to pure matrix parameters, see section 2.2 , implying that its spectral shape remains unchanged.
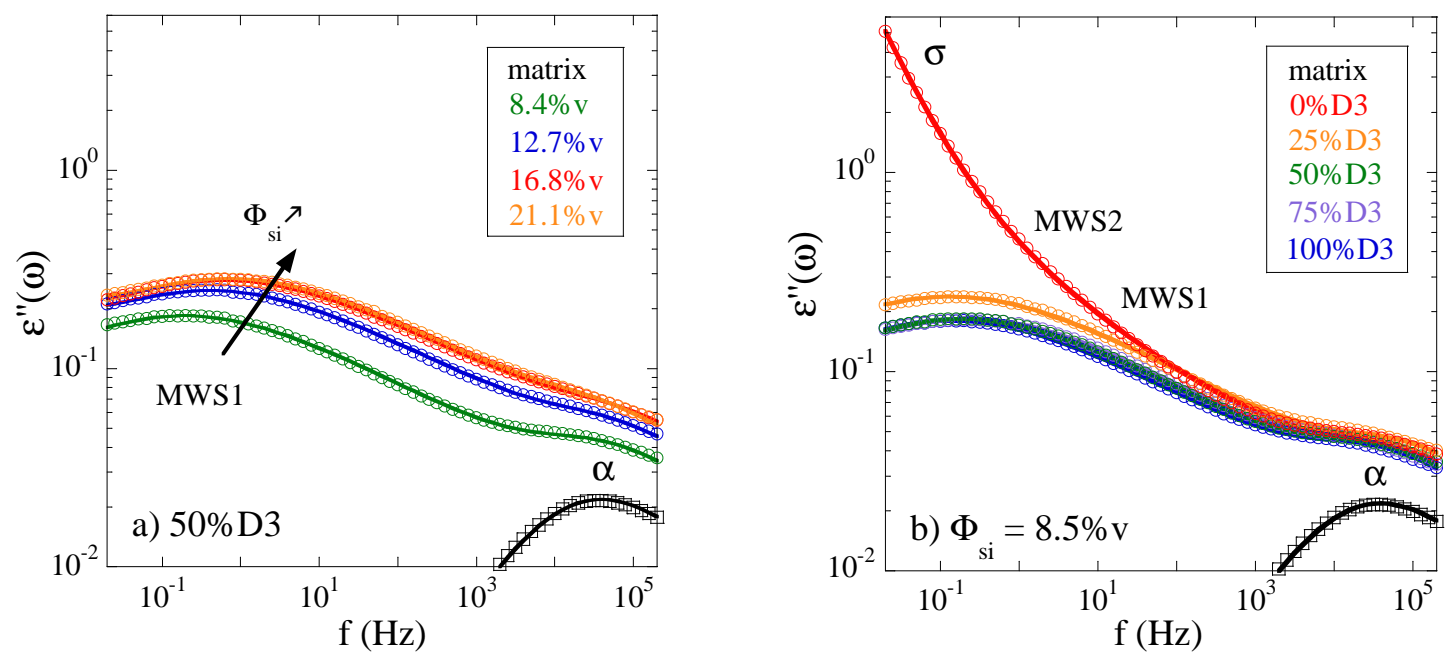

Figure 2: Dielectric loss spectra at $\mathrm{T}=273 \mathrm{~K}$ for nanocomposites with a) different silica volume fractions $\left(\Phi_{\mathrm{si}}=8.4,12.7,16.8\right.$ and $21.1 \% \mathrm{v}$ from bottom to top, 50\%D3), and b) different amounts of graftable chains ( $\% \mathrm{D} 3=0,25,50,75$ and 100 , from top to bottom, $\Phi_{\mathrm{si}} \approx 8.5 \% \mathrm{v}$ ). The matrix signal is shown as black squares. Solid lines are fits to the experimental data by means of eq 1 .

The relaxation times obtained in this way are displayed in Figure 3, where they are compared to those of the matrix. Times for the MWS1-process are given in the SI with a description in terms of the interlayer model $[28,29]$. Within error bars, we observe that the segmental dynamics is not modified by the presence of filler up to the highest silica fraction, in 
agreement with ref [21]. The impact of grafting with $\% \mathrm{D} 3$ from 0 to $100 \%$ is shown in the inset of Figure 3 for $\Phi_{\mathrm{si}} \approx 8.5 \% \mathrm{v}$. There is also no effect of grafting visible in BDS on the segmental dynamics.

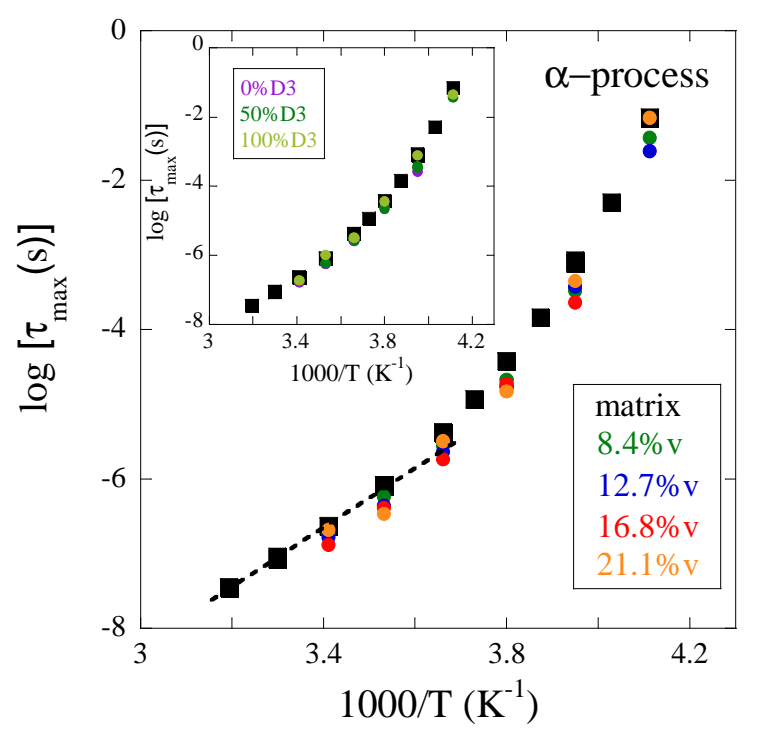

Figure 3: Temperature dependence of the relaxation times for the $\alpha$-process in nanocomposites with $\Phi_{\mathrm{si}}=8.4-21.1 \% \mathrm{v}$ and $50 \% \mathrm{D} 3$. Dotted line is a linear fit of the data above $283 \mathrm{~K}$, see text for details. Inset: same data for different $\% \mathrm{D} 3$ at fixed $\Phi_{\mathrm{si}} \approx 8.5 \% \mathrm{v}$.

The investigation of local segmental dynamics in nanocomposites has been the goal of a large number of works in past years [13-22]. A slowing down of the $\alpha$-process due to interaction or grafting between the polymer chain and the filler has been reported $[14,16,17]$. In this context, distinct contributions have been detected: one part of the chain segments is not affected by the filler, whereas an interfacial polymer layer with frozen dynamics at nanosecond time scales has been evidenced by solid-state NMR experiments, the so-called "glassy" fraction $[13,14]$. Such an immobilized layer with a thickness of 1 to $2 \mathrm{~nm}$ at the particle surface close to $\mathrm{T}_{\mathrm{g}}$ would lead to an increase of the solid-like fraction within the nanocomposite and is thus of utmost importance for filler network percolation [32, 48, 49]. 
We found that the time-scale for the $\alpha$-relaxation is not modified with $\Phi_{\mathrm{si}}$, which means that either there are no immobilized layers, or that BDS is not sensitive enough. To check the existence of an immobilized fraction of organic material (which apart from SB could also comprise the silanization agent octeo) in our nanocomposites, we have performed proton lowfield NMR experiments in the temperature range between 10 and $70^{\circ} \mathrm{C}$. The characteristic time for segmental dynamics as seen by NMR stands in the $\mu$ s range. Thus, the chosen temperatures are just above the "NMR $\mathrm{T}_{\mathrm{g}}$ ", which is roughly located $40 \mathrm{~K}$ above the calorimetric $\mathrm{T}_{\mathrm{g}}[46]$.

Based on eq 3, the apparent rigid polymer fraction $a_{\text {rig }}$ has been determined for the silica fractions between 8.4 and $21.1 \% \mathrm{v}$ using a constant short decay time $\tau_{\text {rig }}=30 \mu$ s as obtained from the DQ measurement. An example of data fitting is shown in Figure 4a, and one can see that there is no rigid contribution in the neat matrix.
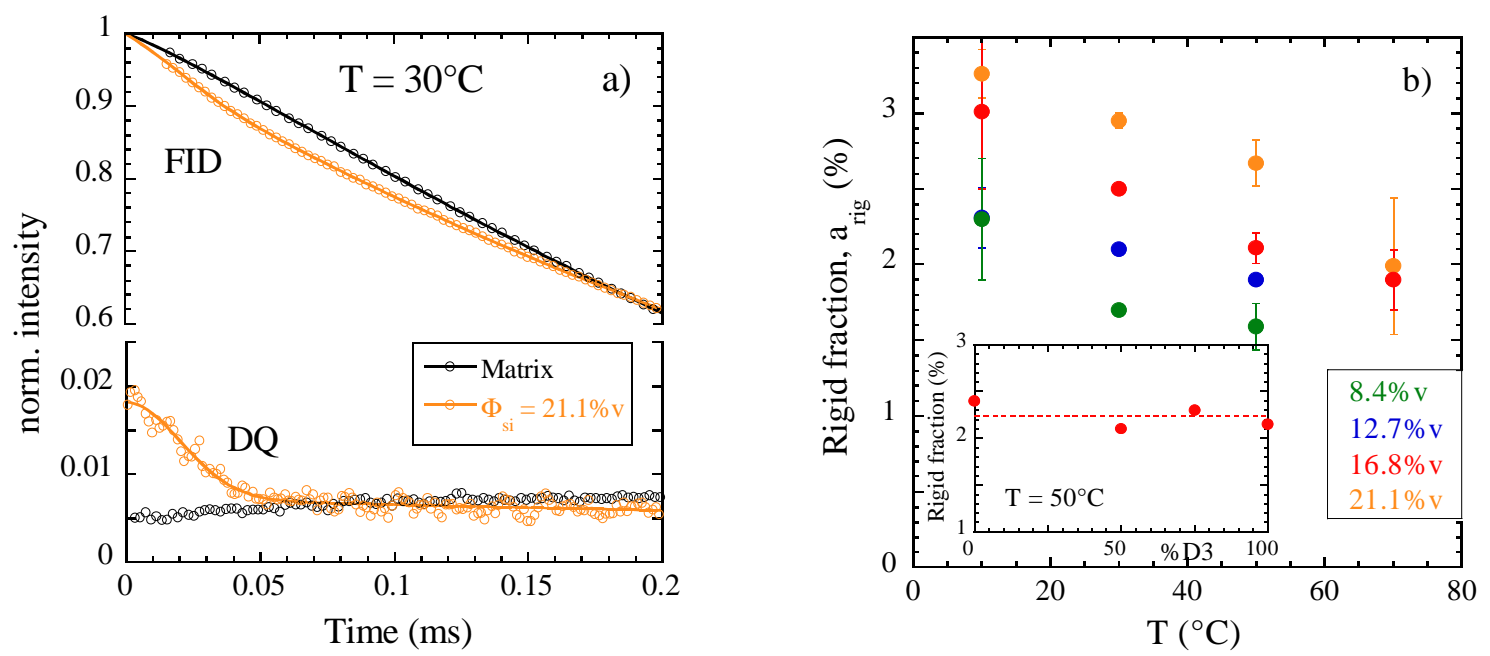

Figure 4: a) FID data at $30^{\circ} \mathrm{C}$ for the matrix and a nanocomposite with $\Phi_{\mathrm{si}}=21.1 \% \mathrm{v}$ and $50 \% \mathrm{D} 3$. The corresponding non-quantitative DQ data used to determine the apparent rigid-fraction time constant, $\tau_{\text {rig }}$, are shown in the lower part. Lines are fits using eq 3. b) NMR-determined fraction of immobilized polymer as a function of temperature for the SB nanocomposites with $\Phi_{\mathrm{si}}=8.4-21.1 \% \mathrm{v}$ and $50 \% \mathrm{D} 3$. Inset: rigid fraction versus $\% \mathrm{D} 3$ at $50^{\circ} \mathrm{C}$ and fixed $\Phi_{\mathrm{si}}=(16.7 \pm 0.1) \% \mathrm{v}$. 
As can be seen in Figure 4b, the apparent amount of rigid polymer increases with $\Phi_{\text {si }}$ and decreases with temperature as expected due to the softening of the immobilized part. The obtained fractions are rather low (between $\approx 1.5$ and $3.5 \%$ ) as compared with other systems like e.g., silica-polyethylacrylate nanocomposites [14]. Note that our results are in agreement with a recent work by Mujtaba et al [32] on industrial SB rubber nanocomposites.

We can rationalize our results based on the structure of aggregates filling fractal branches in our system, cf. [36]. In this paper, we have combined a statistical analysis of TEM pictures with SAXS results and numerical simulations, in order to quantitatively describe the spacefilling properties of silica NPs inside aggregates, and the ones of aggregates inside branches. The main ingredients of structure description in reciprocal space are the NP form factor, the construction of polydisperse aggregates by NPs, the repulsive interactions between aggregates caused by excluded volume and leading to a measurable suppression of the low-q scattering. Finally, the large-scale fractal network is described by a power-law behavior. This SAXS analysis is combined with structural input given by the fraction of branches in the samples obtained by TEM. Aggregates contain typically some 50 primary beads and together with the aggregate radius one can define the internal silica volume fraction termed compacity, $\kappa$. The latter value evolves from 31 to $38 \%$ with $\Phi_{\text {si }}$ in these samples [36], and one can calculate the immobilized polymer fraction per aggregate

$$
\mathrm{a}_{\mathrm{rig}}^{\mathrm{agg}}=\frac{\left(1-\Phi_{\mathrm{si}}\right) \mathrm{a}_{\mathrm{rig}}}{\Phi_{\mathrm{si}}} \frac{\kappa}{1-\kappa}
$$

The latter corresponds to the ratio between the volume of the immobilized polymer and the total polymer volume in one aggregate. At $30^{\circ} \mathrm{C}, \mathrm{a}_{\mathrm{rig}}{ }^{\mathrm{agg}}=(7.3 \pm 0.7) \%$. Within error bars, it is not found to vary with $\Phi_{\text {si }}$. 
A simple estimation of the layer thickness based on the hypothesis of individual bead dispersion gives 0.33 and $0.29 \mathrm{~nm}$ for $\Phi_{\mathrm{si}}=8.4$ and $21.1 \% \mathrm{v}$, respectively, i.e. the immobilized layer is very thin and its thickness independent of $\Phi_{\text {si }}$. This remains also true in presence of small aggregates, like here. Lastly, there was no detectable variation of the immobilized fraction as a function of the quantity of graftable chains (see the insert in Figure $4 \mathrm{~b}$ at fixed $\Phi_{\mathrm{si}} \approx 16.7 \% \mathrm{v}$ ), presumably due to the small number of (end-)grafting functions.

The low fraction of immobilized polymer obtained from NMR confirms the BDS results, where it has been found that the $\alpha$-relaxation was not affected by the presence of silica in our concentration range. Indeed, the resolution of BDS does not allow the detection of the slowdown of such a small polymer fraction.

\subsection{Influence of grafting on percolation}

Grafting of hydrophobic molecules at low grafting density has been shown to affect neither the water-dependent $\beta$ ' (see SI) nor the segmental dynamics ( $\alpha$-process, see Figure 3 ). We now concentrate on the impact of grafting on the high-temperature processes MWS1, MWS2, and the conductivity. The imaginary part of the dielectric permittivity is shown in Figure 5 for samples at fixed silica volume fraction and various fractions of graftable chains in the matrix ranging from $0 \%$ to $100 \% \mathrm{D} 3$ at $333 \mathrm{~K}$. 


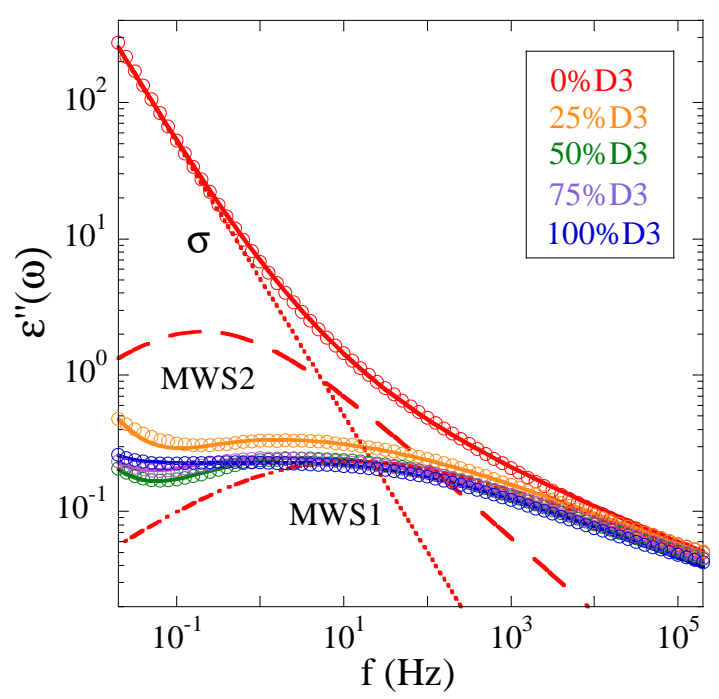

Figure 5: Dielectric loss spectra at $\mathrm{T}=333 \mathrm{~K}$ for nanocomposites with different amounts of graftable chains at fixed $\Phi_{\mathrm{si}} \approx 8.5 \% \mathrm{v}(\% \mathrm{D} 3=0,25,50,75$ and 100 , from top to bottom). Solid lines are fits to the experimental data by means of eq 1 . The fit contributions are included for the sample with $0 \% \mathrm{D} 3$ : the dashed-dotted line stands for the MWS1 contribution, the dashed line for MWS2, and the dotted line for the dc-conductivity.

One notes that the $0 \% \mathrm{D} 3$ sample which contains no graftable chains at all is singular, with a high conductivity and a strong MWS2 contribution (see the detailed fit contributions in the figure), whereas the other samples display much lower values of $\varepsilon^{\prime \prime}(\omega)$ in the low-frequency range. The different MWS-processes can be identified in the broad maximum in Figure 5. They are described by means of the $\mathrm{HN}$ expression using $\Phi_{\mathrm{si}^{-}}$and $\% \mathrm{D} 3$-independent shape parameters (see section 2.2). Their temperature-independent strengths have been averaged from 293 to $363 \mathrm{~K}$ and are plotted as a function of matrix composition \%D3, in Figure 6. 


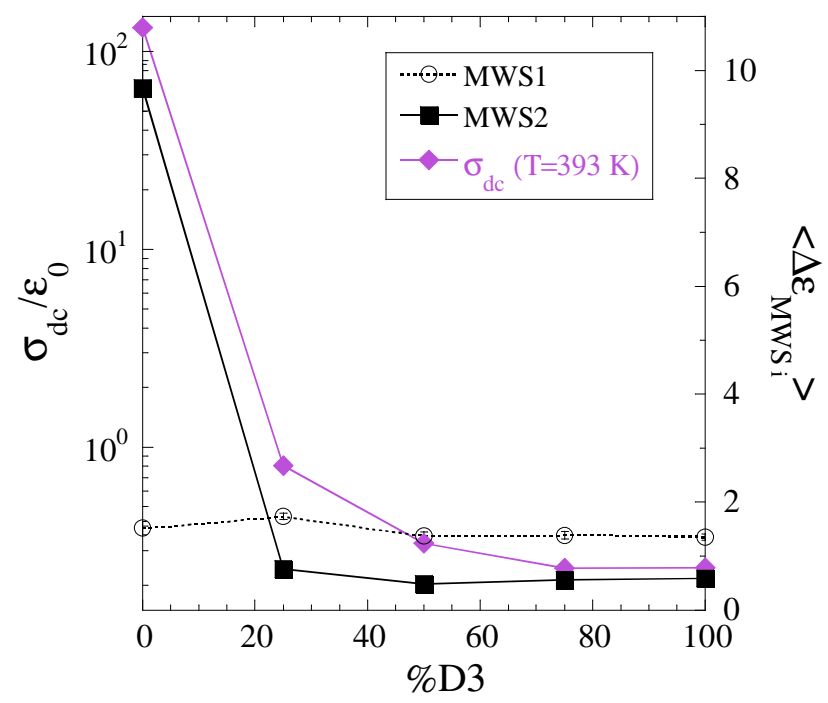

Figure 6: DC-conductivity at $393 \mathrm{~K}$ (diamonds) and average value of the dielectric strengths versus $\mathrm{T}$ for MWS1 (circles) and MWS2 (squares) as a function of the fraction of graftable chains $\left(\Phi_{\mathrm{si}} \approx\right.$ $8.5 \% \mathrm{v})$. Lines are a guide to the eye.

The data in Figure 6 show that the strength of the MWS1 process at fixed $\Phi_{\text {si }}$ is essentially unaffected by grafting as observed for the $\beta$ '-process (see SI). Note that the comparison with the interlayer model $[28,29]$ is favorable, as the strength is determined by the quantity of silica at constant humidity. For both the logarithm of conductivity and dielectric strength of MWS2, there is a continuous decrease with \%D3 leading to saturation. These quantities have been superimposed using the appropriate scale for each, which allows one to see that the shape of the decrease is correlated. MWS1 is found not to be affected by grafting, whereas MWS2 and the logarithm of the conductivity - which show a similar T-dependence - both display the same decrease. This common behavior will be discussed in parallel with the structural and rheological evolution in the next section. To finish, the temperature dependence of the relaxation time of the high-T processes is given in the SI for different matrix compositions, i.e., different grafting, and activation energies are summarized in Table 2. 
Table 2: Activation energy for the dielectric processes MWS1 and MWS2, and the dc-conductivity in nanocomposites with fixed $\Phi_{\mathrm{si}} \approx 8.5 \% \mathrm{v}$ and different fractions of $\% \mathrm{D} 3$.

\begin{tabular}{|r|r|r|r|}
\hline \%D3 & \multicolumn{1}{|c|}{$\mathbf{E}_{\text {MWS1 }}(\mathbf{k J} / \mathbf{m o l})$} & $\mathbf{E}_{\text {Mws2 }}(\mathbf{k J} / \mathbf{m o l})$ & $\mathbf{E}_{\boldsymbol{\sigma}}(\mathbf{k J} / \mathbf{m o l})$ \\
\hline 0 & 58.5 & 63 & 53 \\
\hline 25 & 56 & 121 & 90 \\
\hline 50 & 55 & 120 & 88 \\
\hline 75 & 53 & 108 & 88 \\
\hline 100 & 52 & 107 & 85 \\
\hline
\end{tabular}

As previously observed for $\Phi_{\mathrm{si}}$ variation [40], the MWS2 process displays a similar activation energy as the ionic conductivity and the high-temperature $\alpha$-process $\left(E_{a}=80 \mathrm{~kJ} / \mathrm{mol}\right.$, see dotted line in Figure 3), suggesting again that it is primarily controlled by segmental motion of the polymer matrix via the ionic mobility (diffusion across the matrix).

\section{Discussion of relationship between structure, rheology, and MWS processes.}

The large scale structure as observed by TEM [37] shows the transition from a heterogeneous distribution of silica with large space-spanning branches filled with aggregates at $0 \% \mathrm{D} 3$ to a much more homogeneous distribution of clusters of aggregates at $100 \% \mathrm{D} 3$. This transition with grafting can be described as the depercolation of aggregates, and our dielectric measurements confirm this in a rather unique manner. Indeed, the BDS response in Figure 6 shows that the second interfacial polarization process MWS2 is strongly related to these global structural features. Also, all dielectric processes are associated with a broad distribution of relaxation times, in agreement with structurally polydisperse systems [36].

The depercolation of the large-scale filler structure with increasing polymer grafting has direct consequences on the rheological response of the samples to shear. One of the characteristics of the $G^{\prime}(\omega)$ curves is the plateau modulus $G$, which can also be determined for 
the unfilled matrix $\left(\mathrm{G}_{0}\right)$, allowing to define the reinforcement factor as the ratio of moduli $\mathrm{G} / \mathrm{G}_{0}$. In Figure 7 , we compare the evolution of this reinforcement factor with silica volume fraction of two matrix compositions, $0 \% \mathrm{D} 3$ and $50 \% \mathrm{D} 3$ grafting. The reinforcement was determined at a common frequency of $60 \mathrm{~Hz}$ in raw data taken from refs [36, 50]. Both sets show a weak increase up to some critical percolation volume fraction, and a much stronger one above. However, in the case of the non-functional SB $(0 \% \mathrm{D} 3)$, the critical percolation occurs at lower $\Phi_{\mathrm{si}}$. This illustrates thus the more percolated behavior of the $0 \% \mathrm{D} 3$ at a given volume fraction. For a quantitative comparison, we have fitted the following function [36] to the data

$$
\frac{\mathrm{G}}{\mathrm{G}_{0}}=1+2.5 \Phi_{\mathrm{agg}}+\Theta\left(\Phi_{a g g}-\Phi_{a g g}^{c}\right) \frac{G_{f}}{\mathrm{G}_{0}}\left(\frac{\Phi_{a g g}-\Phi_{a g g}^{c}}{1-\Phi_{a g g}^{c}}\right)^{b}
$$

where $\Phi_{\text {agg }}$ is the volume fraction of aggregates within the branches and defined as $\Phi_{\mathrm{si}} /\left(\kappa \Phi_{\text {fract }}\right)$. Note that average values of the fraction of branches, $\Phi_{\text {fract }}$, have been determined by electron microscopy before $[36,37]$. The fit allows us to determine the relative strength of the network $\mathrm{G}_{\mathrm{f}} / \mathrm{G}_{0}=90(0 \% \mathrm{D} 3)$ and $50(50 \% \mathrm{D} 3)$. The critical silica volume fractions are indicated by arrows in the graph. We obtain $7.5 \pm 0.5 \%$ and $11.5 \pm 0.5 \%$ for $0 \% \mathrm{D} 3$ and $50 \% \mathrm{D} 3$, respectively. This confirms the result of the visual inspection: with a shift of approximately $4 \%$ in volume fraction, this macroscopic technique illustrates that grafting moves the system away from percolation. Such a result agrees nicely with literature results. Indeed, Kumar et al. [51] recently reviewed the different situations arising as a function of the mass and grafting density of chains with respect to matrix chains. Depending on the wetting ability of the polymer brush by the (short or long) polymer matrix chains, different regimes are evidenced leading to very different dispersion of the filler particles in the matrix. In our particular case (same mass of grafted and matrix polymer, high reduced grafting density), grafting induces 
good dispersion, thus shifting the system further away from the percolation threshold as observed in our work.

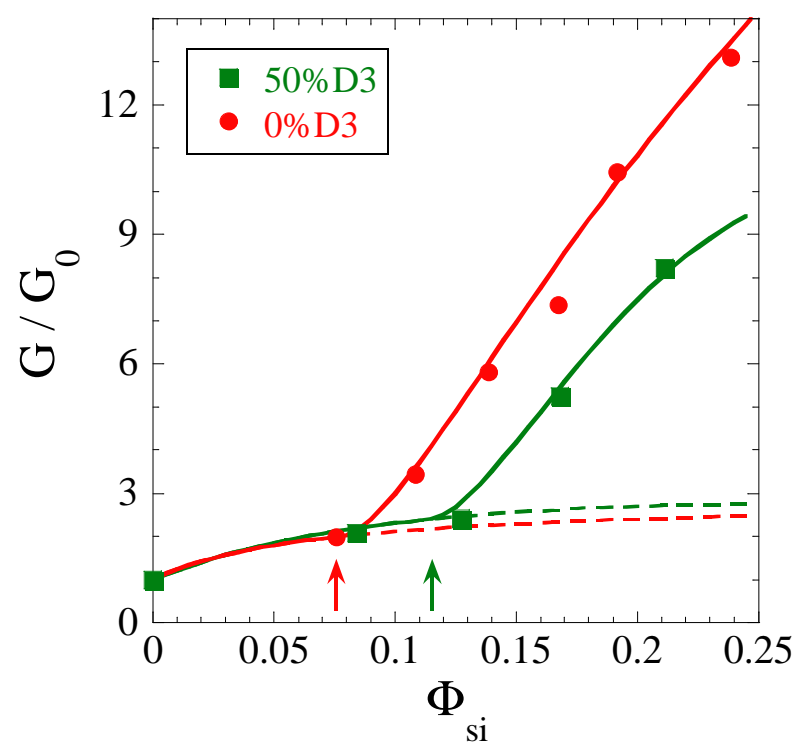

Figure 7: Comparison of rheological response at $60 \mathrm{~Hz}$ in terms of the reinforcement factor $\mathrm{G} / \mathrm{G}_{0}$ as a function of silica volume fraction, for two different grafting matrix compositions, 0\%D3 (circles) and $50 \% \mathrm{D} 3$ (squares). Solid lines are fits with eq 5 . The purely hydrodynamic reinforcement, $1+2.5 \Phi_{\text {agg }}$, is also indicated (dotted lines). Reinforcement values are obtained from data in refs [36, 50].

Incidentally, one may also comment on the NMR results in the light of the reinforcement factors shown in Figure 7. It has been shown recently by Mujtaba et al [32] that reinforcement may be coupled with the fraction of immobilized ("glassy") polymer layers on the surface of the NPs. The interpretation is that even thin layers convey connections between the NPs. These results were based on samples of very similar structure. In the present study, the fraction of (equally thin) immobilized layers was found to be independent of the grafting fraction, which in itself is a surprising result but may be due to the low grafting densities. On the other hand, the corresponding rheological data shown in Figure 7 correlate with the strong structural and dynamical evolution as seen by BDS. It may thus be concluded that the structural contribution - and not the quantity of immobilized polymer - dominates the rheological response in our samples. 


\section{CONCLUSION}

The dielectric properties of silica-filled nanocomposites have been studied by BDS. The insensitivity of the $\alpha$-relaxation to the addition of silica was confirmed by an independent NMR-experiment aiming at the determination of the fraction of an immobilized polymer layer. The equivalent layer thickness was found to be of the order of a few Angstroms, and the "glassy" polymer fraction in an aggregate below $10 \%$. The latter value was determined using the results of a multi-scale structural model based on aggregates of some tens of primary nanoparticles filling large-scale branches and developed by us recently [36]. This calculation thus accounts for the heterogeneous distribution of silica in the matrix, and allows the estimation of the hard fraction of aggregates given by both silica (via aggregate compacity) and immobilized polymer to some $40 \%$. This last value suggests that aggregates can be considered as rigid as compared to the surrounding viscoelastic polymer matrix.

Finally, a second Maxwell-Wagner-Sillars process (MWS2) is shown to evolve strongly with grafting at fixed volume fraction $(8.5 \% \mathrm{v})$. Knowledge of the microstructure and linear rheology of these nanocomposites enables us to correlate these evolutions of dynamics with the structure. Unlike MWS1 but similar to rheology, the MWS2 process is sensitive to depercolation upon polymer grafting. This establishes that dielectric spectroscopy can be used as a simultaneous probe for large-scale structural reorganization in complex silica-polymer nanocomposites and local dynamics. In combination with NMR, it shows that structural contributions to the rheological response dominate those of immobilized polymer in our samples.

Acknowledgements: We are thankful for a "Chercheur d'Avenir" grant by the LanguedocRoussillon region (J.O.) and Ph.D. funding "CIFRE" by Michelin (G.P.B.). The authors acknowledge financial support from the European Commission under the Seventh Framework 
Program by means of the grant agreement for the Integrated Infrastructure Initiative N. 262348 European Soft Matter Infrastructure (ESMI). We are indebted to C. Dupas (L2C) and A. Mujtaba (Halle) for experimental support.

\section{References}

[1] J. Jancar, J. F. Douglas, F. W. Starr, S. K. Kumar, P. Cassagnau, A. J. Lesser, S. S. Sternstein, M. J. Buehler. Current issues in research on structure-property relationships in polymer nanocomposites. Polymer 2010;51:3321-3343.

[2] G. Heinrich, M. Kluppel, T. A. Vilgis. Reinforcement of elastomers. Current Opinion in Solid State \& Materials Science 2002;6:195-203.

[3] J. E. Mark, B. Erman, F. R. Eirich, Science and Technology of Rubber, Academic Press, San Diego, 1994.

[4] G. Schmidt, M. M. Malwitz. Properties of polymer-nanoparticle composites. Current Opinion in Colloid \& Interface Science 2003;8:103-108.

[5] I. Armentano, M. Dottori, E. Fortunati, S. Mattioli, J. M. Kenny. Biodegradable polymer matrix nanocomposites for tissue engineering: A review. Polym. Degrad. Stabil. 2010;95:2126-2146.

[6] J. Oberdisse. Aggregation of colloidal nanoparticles in polymer matrices. Soft Matter 2006;2:29-36.

[7] N. Jouault, P. Vallat, F. Dalmas, S. Said, J. Jestin, F. Boue. Well-Dispersed Fractal Aggregates as Filler in Polymer-Silica Nanocomposites: Long-Range Effects in Rheology. Macromolecules 2009;42:2031-2040.

[8] G. Capuano, G. Filippone, G. Romeo, D. Acierno. Universal Features of the Melt Elasticity of Interacting Polymer Nanocomposites. Langmuir 2012;28:5458-5463. 
[9] M. I. Aranguren, E. Mora, J. V. Degroot, C. W. Macosko. Effect of reinforcing fillers on the rheology of polymer melts. Journal of Rheology 1992;36:1165-1182.

[10] S. S. Choi, B. H. Park, H. Song. Influence of filler type and content on properties of styrene-butadiene rubber (SBR) compound reinforced with carbon black or silica. Polymers for Advanced Technologies 2004;15:122-127.

[11] A. R. Payne, R. E. Whitaker. Low strain dynamic properties of filled rubbers. Rubber Chem Technol 1971;44:440-478.

[12] H. Montes, T. Chaussee, A. Papon, F. Lequeux, L. Guy. Particles in model filled rubber: Dispersion and mechanical properties. European Physical Journal E 2010;31:263-268.

[13] J. Berriot, H. Montes, F. Lequeux, D. Long, P. Sotta. Evidence for the shift of the glass transition near the particles in silica-filled elastomers. Macromolecules 2002;35:97569762.

[14] A. Papon, K. Saalwächter, K. Schäler, L. Guy, F. Lequeux, H. Montes. Low-Field NMR Investigations of Nanocomposites: Polymer Dynamics and Network Effects. Macromolecules 2011;44:913-922.

[15] J. H. Roh, M. Tyagi, T. E. Hogan, C. M. Roland. Space-Dependent Dynamics in 1,4Polybutadiene Nanocomposite. Macromolecules 2013;46:6667-6669.

[16] D. Fragiadakis, L. Bokobza, P. Pissis. Dynamics near the filler surface in natural rubber-silica nanocomposites. Polymer 2011;52:3175-3182.

[17] V. Arrighi, I. J. McEwen, H. Qian, M. B. S. Prieto. The glass transition and interfacial layer in styrene-butadiene rubber containing silica nanofiller. Polymer 2003;44:62596266. 
[18] R. H. Gee, R. S. Maxwell, B. Balazs. Molecular dynamics studies on the effects of water speciation on interfacial structure and dynamics in silica-filled PDMS composites. Polymer 2004;45:3885-3891.

[19] M. Klüppel. Evaluation of viscoelastic master curves of filled elastomers and applications to fracture mechanics. J. Phys.-Condes. Matter 2009;21:035104

[20] S. Fotiadou, C. Karageorgaki, K. Chrissopoulou, K. Karatasos, I. Tanis, D. Tragoudaras, B. Frick, S. H. Anastasiadis. Structure and Dynamics of Hyperbranched Polymer/Layered Silicate Nanocomposites. Macromolecules 2013;46:2842-2855.

[21] J. Otegui, G. A. Schwartz, S. Cerveny, J. Colmenero, J. Loichen, S. Westermann. Influence of Water and Filler Content on the Dielectric Response of Silica-Filled Rubber Compounds. Macromolecules 2013;46:2407-2416.

[22] V. M. Boucher, D. Cangialosi, A. Alegria, J. Colmenero, J. Gonzalez-Irun, L. M. LizMarzan. Accelerated physical aging in PMMA/silica nanocomposites. Soft Matter 2010;6:3306-3317.

[23] A. Papon, H. Montes, F. Lequeux, J. Oberdisse, K. Saalwachter, L. Guy. Solid particles in an elastomer matrix: impact of colloid dispersion and polymer mobility modification on the mechanical properties. Soft Matter 2012;8:4090-4096.

[24] J. Mijovic, H. K. Lee, J. Kenny, J. Mays. Dynamics in polymer-silicate nanocomposites as studied by dielectric relaxation spectroscopy and dynamic mechanical spectroscopy. Macromolecules 2006;39:2172-2182.

[25] M. Bohning, H. Goering, A. Fritz, K. W. Brzezinka, G. Turky, A. Schonhals, B. Schartel. Dielectric study of molecular mobility in poly(propylene-graft-maleic anhydride)/clay nanocomposites. Macromolecules 2005;38:2764-2774. 
[26] Y.-H. Lee, A. J. Bur, S. C. Roth, P. R. Start. Accelerated $\alpha$ Relaxation Dynamics in the Exfoliated Nylon-11/Clay Nanocomposite Observed in the Melt and Semicrystalline State by Dielectric Spectroscopy. Macromolecules 2005;38:3828-3837.

[27] J. G. Meier, M. Kluppel. Carbon black networking in elastomers monitored by dynamic mechanical and dielectric spectroscopy. Macromolecular Materials and Engineering 2008;293:12-38.

[28] P. A. M. Steeman, F. H. J. Maurer. AN INTERLAYER MODEL FOR THE COMPLEX DIELECTRIC-CONSTANT OF COMPOSITES. Colloid Polym. Sci. 1990;268:315325.

[29] P. A. M. Steeman, F. H. J. Maurer, M. A. Vanes. DIELECTRIC MONITORING OF WATER-ABSORPTION IN GLASS-BEAD-FILLED HIGH-DENSITY POLYETHYLENE. Polymer 1991;32:523-530.

[30] J. Fritzsche, M. Kluppel. Structural dynamics and interfacial properties of fillerreinforced elastomers. Journal of Physics-Condensed Matter 2011;23.

[31] H. Montes, F. Lequeux, J. Berriot. Influence of the glass transition temperature gradient on the nonlinear viscoelastic behavior in reinforced elastomers. Macromolecules $2003 ; 36: 8107-8118$

[32] A. Mujtaba, M. Keller, S. Ilisch, H. J. Radusch, M. Beiner, T. Thurn-Albrecht, K. Saalwächter. Detection of Surface-Immobilized Components and Their Role in Viscoelastic Reinforcement of Rubber-Silica Nanocomposites. ACS Macro Letters $2014 ; 3: 481-485$.

[33] P. Sotta, D. Long. The crossover from 2D to 3D percolation: Theory and numerical simulations. European Physical Journal E 2003;11:375-387.

[34] J. Oberdisse. Structure and rheological properties of latex-silica nanocomposite films: Stress-strain isotherms. Macromolecules 2002;35:9441-9450. 
[35] J. Oberdisse, B. Deme. Structure of latex-silica nanocomposite films: A small-angle neutron scattering study. Macromolecules 2002;35:4397-4405.

[36] G. P. Baeza, A. C. Genix, C. Degrandcourt, L. Petitjean, J. Gummel, M. Couty, J. Oberdisse. Multiscale Filler Structure in Simplified Industrial Nanocomposite Silica/SBR Systems Studied by SAXS and TEM. Macromolecules 2013;46:317-329.

[37] G. P. Baeza, A. C. Genix, C. Degrandcourt, L. Petitjean, J. Gummel, R. Schweins, M. Couty, J. Oberdisse. Effect of Grafting on Rheology and Structure of a Simplified Industrial Nanocomposite Silica/SBR. Macromolecules 2013;46:6388-6394.

[38] G. P. Baeza, A. C. Genix, C. Degrandcourt, J. Gummel, A. Mujtaba, K. Saalwachter, T. Thurn-Albrecht, M. Couty, J. Oberdisse. Studying twin-samples provides evidence for a unique structure-determining parameter in simplified industrial nanocomposites. ACS Macro Letters 2014;3:448-452.

[39] G. P. Baeza, A. C. Genix, C. Degrandcourt, J. Gummel, M. Couty, J. Oberdisse. Mechanism of aggregate formation in simplified industrial silica styrene-butadiene nanocomposites: effect of chain mass and grafting on rheology and structure. Soft Matter 2014;10:6686-6695.

[40] G. P. Baeza, J. Oberdisse, A. Alegria, M. Couty, A. C. Genix. A high-temperature dielectric process as a probe of large-scale silica filler structure in simplified industrial nanocomposites. Phys. Chem. Chem. Phys. 2015;17:1660-1666.

[41] S. Cerveny, R. Bergman, G. A. Schwartz, P. Jacobsson. Dielectric $\alpha$ - and $\beta$-Relaxations in Uncured Styrene Butadiene Rubber. Macromolecules 2002;35:4337-4342.

[42] S. Cerveny, G. A. Schwartz, J. Otegui, J. Colmenero, J. Loichen, S. Westermann. Dielectric Study of Hydration Water in Silica Nanoparticles. Journal of Physical Chemistry C 2012;116:24340-24349. 
[43] F. Kremer, A. Schönhals, Broadband Dielectric Spectroscopy, Springer-Verlag, Heidelberg, 2003.

[44] C. A. Angell. RELAXATION IN LIQUIDS, POLYMERS AND PLASTIC CRYSTALS - STRONG FRAGILE PATTERNS AND PROBLEMS. Journal of NonCrystalline Solids 1991;131:13-31.

[45] J. D. Ferry, Viscoelastic properties of polymers, John Wiley and Sons, New York, 1980.

[46] K. Saalwächter. MICROSTRUCTURE AND MOLECULAR DYNAMICS OF ELASTOMERS AS STUDIED BY ADVANCED LOW-RESOLUTION NUCLEAR MAGNETIC RESONANCE METHODS. Rubber Chemistry and Technology 2012;85:350-386.

[47] M. Mauri, Y. Thomann, H. Schneider, K. Saalwächter. Spin-diffusion NMR at low field for the study of multiphase solids. Solid State Nuclear Magnetic Resonance 2008;34:125-141.

[48] S. Merabia, P. Sotta, D. R. Long. A Microscopic Model for the Reinforcement and the Nonlinear Behavior of Filled Elastomers and Thermoplastic Elastomers (Payne and Mullins Effects). Macromolecules 2008;41:8252-8266.

[49] A. Mujtaba, M. Keller, S. Ilisch, H. J. Radusch, T. Thurn-Albrecht, K. Saalwachter, M. Beiner. Mechanical Properties and Cross-Link Density of Styrene-Butadiene Model Composites Containing Fillers with Bimodal Particle Size Distribution. Macromolecules 2012;45:6504-6515.

[50] A. Bouty, L. Petitjean, C. Degrandcourt, J. Gummel, P. Kwaśniewski, F. Meneau, F. Boué, M. Couty, J. Jestin. Nanofiller Structure and Reinforcement in Model Silica/Rubber Composites: A Quantitative Correlation Driven by Interfacial Agents. Macromolecules 2014;47:5365-5378. 
[51] S. K. Kumar, N. Jouault, B. Benicewicz, T. Neely. Nanocomposites with Polymer Grafted Nanoparticles. Macromolecules 2013;46:3199-3214. 